\title{
A META-ANALYSIS OF DRUG RESISTANT TUBERCULOSIS IN SUB-SAHARAN AFRICA: HOW STRONGLY ASSOCIATED WITH PREVIOUS TREATMENT AND HIV CO-INFECTION?
}

\author{
Asres Berhan', Yifru Berhan', Desalegn Yizengaw ${ }^{2}$
}

ABSTRACT

BACKGROUND: In Sub-Saharan Africa, the fight against tuberculosis (TB) has encountered a great challenge because of the emergence of drug resistant TB strains and the high prevalence of $\mathrm{HIV}$ infection. The aim of this meta-analysis was to determine the association of drug-resistant TB with antiTB drug treatment history and HIV co-infection.

METHODS: After electronic based literature search in the databases of Medline, HINARI, EMBASE and the Cochrane library, article selection and data extraction were carried out. HIV co-infection and previous history of TB treatment were used as predictors for the occurrence of any anti-TB drug resistant or multiple drug resistant TB (MDR-TB). The risk ratios for each included study and for the pooled sample were computed using the random-effects model. Heterogeneity test, sensitivity analyses and funnel plots were also done.

RESULTS: The pooled analysis showed that the risk of developing drug-resistant TB to at least one antiTB drug was about 3 times higher in individuals who had a previous history of anti-TB treatment than new TB cases. The risk of having MDR-TB in previously anti-TB treated TB cases was more than 5-fold higher than that of new TB cases. Resistance to Ethambutol and Rifampicin was more than fivefold higher among the previously treated with anti-TB drugs. However, HIV infection was not associated with drug-resistant $\mathrm{TB}$.

CONCLUSION: There was a strong association of previous anti-TB treatment with MDR-TB. Primary treatment warrants special emphasis, and screening for anti-TB drugs sensitivity has to be strengthened. KEYWORDS: drug resistance, HIV, meta-analysis, previous treatment, tuberculosis, Sub-Saharan Africa

DOI: http://dx.doi.org/10.4314/ejhs.v23i3.10

\section{INTRODUCTION}

Tuberculosis (TB) and HIV co-infection continues to be one of the major public health problems in Sub-Saharan Africa (SSA). Since 1990, the number of people falling ill and dying of TB has been declining very slowly (1). But among infectious diseases, following HIV infection, TB is still the second leading cause of death worldwide and the first leading cause of death among HIV infected patients (2). In African region, the number of deaths caused by TB (including deaths of HIV-positive people) in the year 2010 was estimated to be 254, 000 (2).

Since TB and HIV infections interact synergistically (3), the efforts to alleviate morbidities and mortalities due to TB have been facing challenges. The presence of HIV infection in TB infected persons (asymptomatic) accelerates the chance of developing TB disease and similarly, TB hastens HIV disease advancement (3).

\footnotetext{
${ }^{1}$ College of Medicine and Health Sciences, Hawasa Univeristy, Ethiopia

${ }^{2}$ University of Manitoba, Canada

Corresponding Author: Yifru Berhan, Email:yifrub@yahoo.com
} 
In 2010, from the 8.8 million incidences of TB cases, about $13 \%$ were HIV co-infected; of HIV co-infected, the African region alone contributed for $82 \%$ (2). Besides HIV, the fight against TB has encountered a great challenge by the widely spreading and emerging drug resistant TB infections (4). Globally, in 2010, 3.4\% of new TB cases were estimated to be multidrug resistant (MDR-TB) (4). Though the number of surveys was small, data from some SSA countries indicated that the proportion of resistant TB (resistant to any anti-TB drugs or MDR) was lower than in Eastern Europe and some Asian countries, but South Africa was an outlier (5).

Resistant TB strains can be acquired or new resistant TB strains can emerge due to different factors: previous use of quinolones (6), inappropriate TB treatment (7), poor adherence to anti-TB drugs, long-lasting illnesses, previous TB treatment $(8,9)$. Very recently, WHO concluded that people living with HIV are facing the emerging threats of drug resistant TB (4), which was also noted in another report (10). Nevertheless, most studies emphasized the role of HIV and previous TB treatment in the development of drug resistant TB infection.

The existing primary studies in SSA that assessed the association of anti-TB drug resistant TB with HIV lack consistency: some studies showed association of drug-resistant TB with HIV infection (11-13) while others reported no association with HIV (14-20). Additionally, in 2008, a multi-study analysis that included seven SSA studies (all conducted before the year 2000) could not demonstrate an overall association of drug-resistant TB with HIV infection (21). However, the majority of the studies that assessed the association of drug-resistant TB with previous history of anti-TB drug treatment reported a statistically significant association $(15-17,20,22)$.

To date, there is no published meta-analysis that included the most recent studies exclusively in SSA, which have assessed the association of HIV and anti-TB drug treatment history with drug-resistant TB. Thus, the primary aim of this meta-analysis is to determine how strongly drugresistant TB was associated with anti-TB drug treatment history and HIV co-infection.

\section{METHODS}

Search strategy: After the authors reached agreement on the objectives and the possible search terms to be used, a computer based literature search was conducted by two of the authors independently. Databases that were searched include: Medline, HINARI, EMBASE and the Cochrane library. The search was further strengthened by searching any missed literatures with Google scholar search engine and by searching the reference list of relevant articles. Our search terms include: "Resistant TB", "MDRTB", "XDR-TB", "HIV", "Africa", "Sub-Saharan Africa" and the names of Sub-Saharan African countries.

Inclusion criteria and study selection: The predetermined inclusion criteria for this metaanalysis were: 1) studies conducted in SubSaharan Africa, 2) either longitudinal/cohort or cross-sectional studies which assessed the association of anti-TB drug resistant tuberculosis with HIV and/or anti-TB drug treatment history and 3) studies written in English. These criteria were set because the aim of this study was to assess the association of drug resistant $\mathrm{TB}$ with previous anti-TB drug exposure and HIV infection.

Article selection was performed in two stages: first, after the titles and abstracts of the retrieved articles were reviewed, they were grouped as "likely" and "unlikely". Second, the contents of articles grouped as "likely" by both authors and articles that were not selected by both authors (one author grouped them as "likely" and the other as "unlikely") were reviewed in detail before the final decisions. If the two reviewers were still unable to decide whether to include or exclude a particular study, the conflict was resolved by a discussion involving the third author.

Data extraction: Excel spreadsheet was used by both authors to extract the data. The extracted data included: author, year of publication, event time, country where the study was conducted, total number of HIV positives, number of HIV positives co-infected with any drug resistant TB, number of HIV positives co-infected with MDRTB, total number of HIV negatives, number of HIV negatives infected with any anti-TB drug resistant $\mathrm{TB}$, number of HIV negatives infected with MDR-TB, total number of TB infected individuals with no previous history of anti-TB drug treatment, number of any anti-TB drug resistant TB infected individuals with no previous 
history of anti-TB drug treatment, number of MDR-TB infected individuals with no previous history of anti-TB drug treatment, total number of TB infected individuals with previous history of anti-TB drug treatment, number of any anti-TB drug resistant $\mathrm{TB}$ infected individuals with previous history of anti-TB drug treatment and number of MDR-TB infected individuals with previous history of anti-TB treatment.

Operational definitions: In this meta-analysis, we used the term "any anti-TB drug resistance TB" to mean resistant TB to one or more anti-TB drugs. "MDR-TB" means that TB is resistant to at least isoniazid and rifampicin (1). Those grouped as "Individuals with previous history of anti-TB drug treatment" include: relapse, failures and return to treatment. In the meta-analysis that compared new and previously anti-TB drug treated cases, their HIV status could be either negative or positive. Similarly, in HIV positives and negatives TB drug-resistance comparison, the included individuals can be new or previously treated TB cases.

Statistical analysis: The associations of any drugresistant TB and MDR-TB with HIV and anti-TB drug treatment history were meta-analyzed separately (anti-TB drug resistant TB in HIV positives vs anti-TB drug resistant TB in HIV negatives; MDR-TB in HIV positives vs MDR-TB in HIV negatives; any anti-TB drug resistant TB in previously anti-TB treated vs any anti-TB drug resistant $\mathrm{TB}$ in new $\mathrm{TB}$ cases and MDR-TB in previously anti-TB drug treated vs MDR-TB in new TB cases). Meta-analysis was also done for individual first line anti-TB drugs in relation to TB treatment history and HIV status. The overall risk ratios were determined with the DerSimonianLaird method (random-effects model).

The heterogeneity among the studies was assessed by computing values for chi-square (Q), $\mathrm{I}^{2}$ and $\mathrm{p}$-values. $\mathrm{I}^{2} \geq 50 \%$ was considered as statistically significant. Since the included studies were conducted with different setups, and because patients differed in their experience for antiretroviral therapy, we preferred to report the results of random effects model even in cases of insignificant heterogeneity. Furthermore, some patients who were grouped as anti-TB experienced could be cases of reinfection or relapse cases that will increase the heterogeneity of the included studies.

To reveal the change in drug resistant $\mathrm{TB}$ with time, sub-group analyses based on study periods were carried out (studies before the year $2000 v s$ studies after 2000). Sensitivity analyses (leaving one study out at a time) were conducted to estimate the stability of the overall risk ratios in the withdrawal of any studies from the analysis. Publication/disclosure biases were assessed with funnel plots. All the statistical analyses and plots were generated using Meta Analyst (Beta 3.13) software.

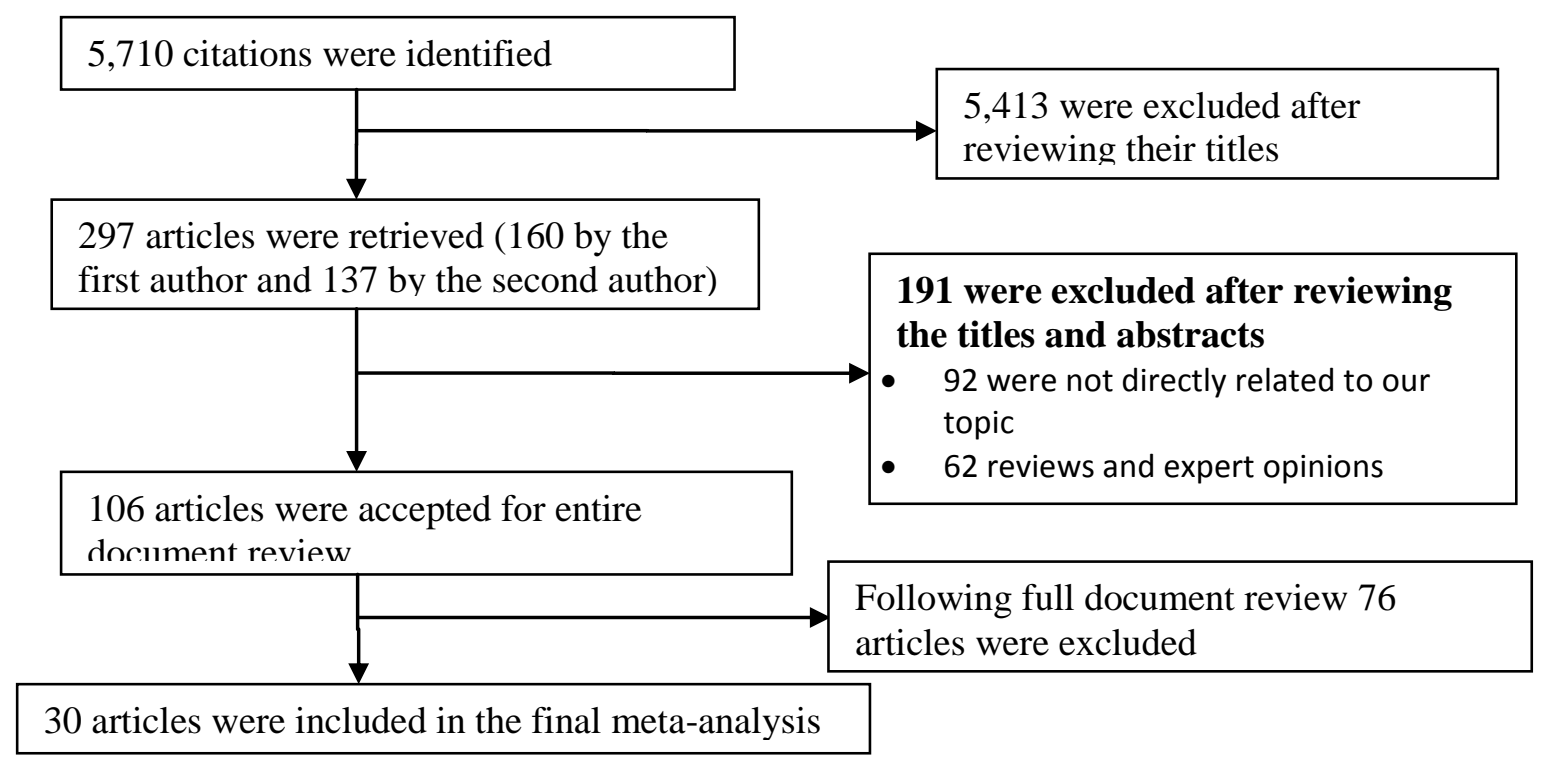

Figure 1. Flow diagram to show study selection process, Sub-Saharan Africa 


\section{RESULTS}

Search Result: As summarized in Fig.1, the literature search with the chosen search terms initially identified 5,710 citations. Taking the articles' title proximity to the objective of this study, 297 articles were retrieved. After rescreening the titles and reviewing the abstracts, 106 of the retrieved articles were accepted for detailed document review. Finally, 30 articles met the inclusion criteria for this meta-analysis (11-20, 22, 23-41). However, all the selected articles were not included in every meta-analysis. The age range of study participants in the included studies was 11 years -86 years.

Study Name

Demissie M et al (2001)

Kenyon A T (1999)

Yimer S A et al (2012)

Abebe $G$ et al (2012)

Lawson $L$ et at (2010)

Urassa W et a (2008)

Sangare $L$ et al study 1 (2010)

Asiimwe B B et al (2008)

Diandé $S$ ot al (2009)

Bruchfeld J eta al (2002)

van Halsema C L et al (2012)

Murray $J$ et al study 2 (1999)

Mac-Arthur A ot al (2001)

Bercion R ot al (1997)

Anastasis D et al (1997)

Murray $J$ et al study 1 (2000)

Davies GR ot al (1999)

Sangaré L et al study 2 (2011)

Lukoye D et al (2011)

Minime-Lingoupou F et al (2011)

Overall

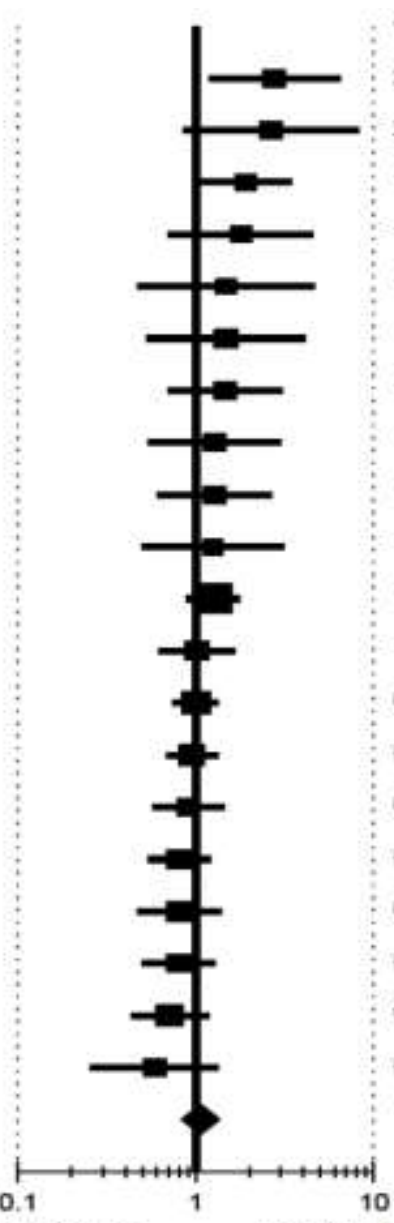

Any anti-TB resistance in HIV positives
HIV Infection and Drug Resistance TB: Specifically to determine the association of HIV infection with drug resistant TB meta-analysis, only 20 articles were included (with overall 3,371 HIV infected and 3,937 HIV negative individuals). With the exception of two Ethiopian studies (11, 12), all other studies showed no association of any anti-TB drug resistance to either HIV positives or HIV negatives, and the overall risk ratio (RR) was 1.1 (95\% CI, 0.92 to 1.23 ) (Fig 2). Sensitivity analysis attested the stability of the overall risk ratio; the overall RR swings between 1.01 and 1.06. The test for heterogeneity did not show a significant variation among the twenty studies $\left(\mathrm{I}^{2}\right.$ $=23 \%$ ). Likewise, the funnel plots did not show the existence of publication/disclosure biases.

$\begin{array}{lc}\text { Confidence Interval } & \text { Country } \\ 2.756(1.177,6.450) & \text { Ethiopia } \\ 2.628(0.848,8.149) & \text { Botswana } \\ 1.920(1.075,3.428) & \text { Ethiopia } \\ 1.785(0.700,4.552) & \text { Ethiopia } \\ 1.474(0.470,4.623) & \text { Nigeria } \\ 1.474(0.525,4.137) & \text { Tanzania } \\ 1.454(0.692,3.055) & \text { Burkina Faso } \\ 1.275(0.541,3.009) & \text { Uganda } \\ 1.268(0.609,2.639) & \text { Burkina Faso } \\ 1.248(0.494,3.150) & \text { Ethiopia } \\ 1.239(0.876,1.752) & \text { South Africa } \\ 1.011(0.614,1.665) & \text { South Africa } \\ 0.995(0.736,1.344) & \text { Mozambique } \\ 0.949(0.676,1.331) & \text { Cameroon } \\ 0.907(0.574,1.432) & \text { South Africa } \\ 0.808(0.535,1.220) & \text { South Africa } \\ 0.807(0.465,1.403) & \text { South Africa } \\ 0.795(0.496,1.274) & \text { Curkina Faso } \\ 0.711(0.431,1.173) & 061(0.918,1.226)\end{array}$

Any anti-TB resistance in HIV negatives

Heterogeneity test

\begin{tabular}{cccc}
\hline \multicolumn{4}{c}{ Heterogeneity test } \\
\hline $1^{2}$ & $Q$ & DF & P-val (Q) \\
$23 \%$ & 24.663 & 19.000 & 0.172 \\
\hline
\end{tabular}

Figure 2. Risk ratio of any anti-TB drug resistance in relation to HIV status (HIV positives vs HIV negatives), a metaanalysis in Sub-Saharan Africa. 
HIV Infection and MDR TB: The meta-analysis of MDR-TB did not also demonstrate a statistically significant association of MDR-TB with HIV co-infection. As presented in Fig.3, except for two studies (from Swaziland and Mozambique) (23, 33), all other studies showed the association of MDR-TB to HIV positives or
HIV negatives with an overall RR of 1.0 and $95 \%$ CI, 0.78 to 1.36 . When any of the studies were withdrawn from the analysis, the overall risk ratio ranged between 0.94 and 1.06.

Heterogeneity testing showed only a moderate degree of variation among the studies $\left(\mathrm{I}^{2}\right.$ $=32 \%$ ).

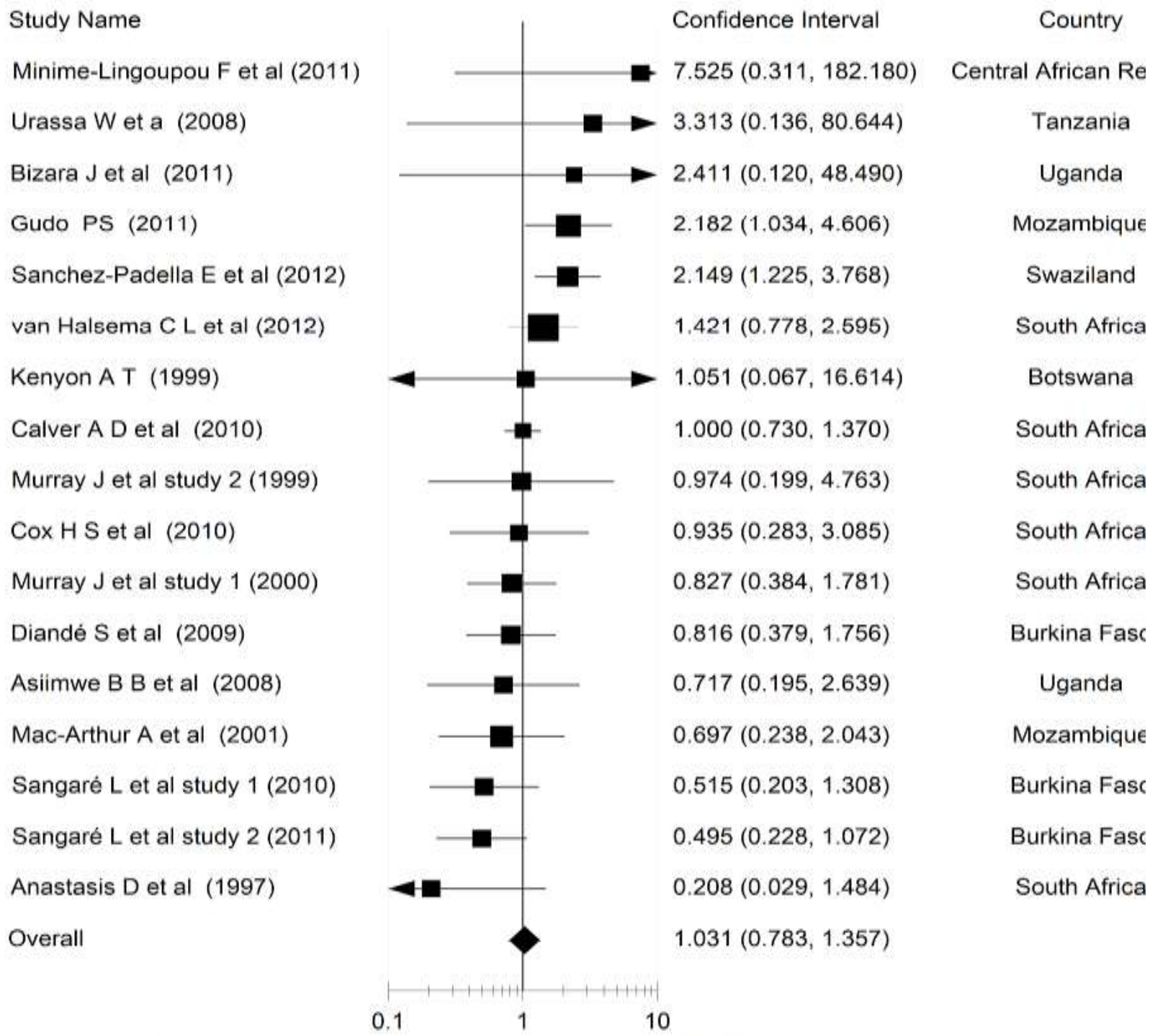

MDR TB in HIV negatives

MDR TB in HIV positives

\begin{tabular}{cccc}
\hline \multicolumn{4}{c}{ Heterogeniety test } \\
\hline $\mathrm{I}^{2}$ & $\mathrm{Q}$ & $\mathrm{DF}$ & $\mathrm{P}$-val (Q) \\
$31.7 \%$ & 23.436 & 16.000 & 0.103 \\
\hline
\end{tabular}

Figure 3. Risk ratio of multidrug resistant TB (MDR-TB) in relation to HIV status (HIV positives vs HIV negatives), a meta-analysis in Sub-Saharan Africa. 
Previous Anti-TB Treatment and Drug Resistant TB: However, drug resistant TB was found to be significantly associated with a previous history of anti-TB drug treatment. The risk of having TB that was resistant to at least one anti-TB drug was about 3 times higher in individuals with a previous history of anti-TB treatment. As shown in Fig 4, with the exception of the studies by Nunes et al, Mulenga et al, and Bruchfeld et al $(22,31,36)$, all others (with an overall RR of 2.9 and $95 \% \mathrm{CI}, 2.38$ to 3.46 ) demonstrated a significant association of anti-TB drug resistance with a previous history of exposure to anti-TB drugs. But heterogeneity testing revealed the presence of a significant variation among the studies $\left(\mathrm{I}^{2}=72 \%\right)$. Sub-group analysis based on the period of data collection (before 2000 vs after 2000) did not show a significant variation in anti-TB drug resistance. Sensitivity analysis showed that the overall RR changed by about 0.1 at maximum with the withdrawal of any of the included studies.
Study Name

Sangaré L et al study 2 (2011)

Cox HS et al (2010)

Sangaré L et al study 1 (2010)

Kenyon A T (1999)

Murray $\mathrm{J}$ et al study 1 (2000)

Sanchez-Padella E et al (2012)

Chonde TM et al (2010)

van Halsema C L et al (2012)

Lukoye D et al (2011)

Gudo PS (2011)

Mac-Arthur A et al (2001)

Affolabi D et al (2007)

Umubyeyi AN et al (2007)

Mulenga $C$ et al (2010)

Davies GR et al (1999)

Nunes E A et al (2005)

Bruchfeld J eta al (2002)

Overall

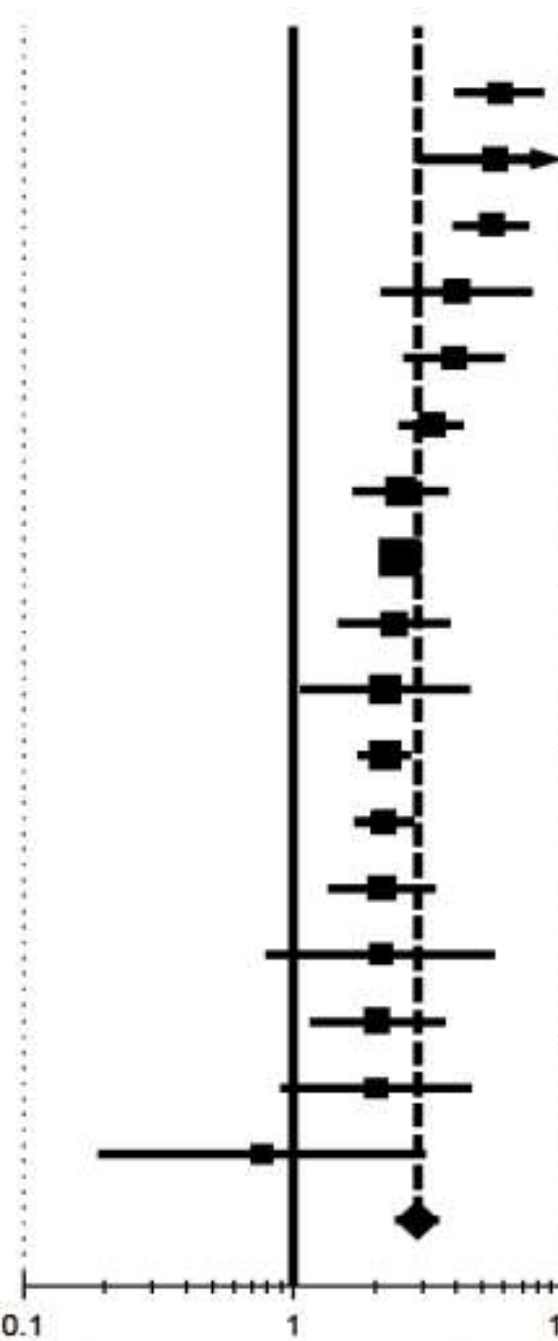

Any anti-TB resistance in previously anti-TB treated

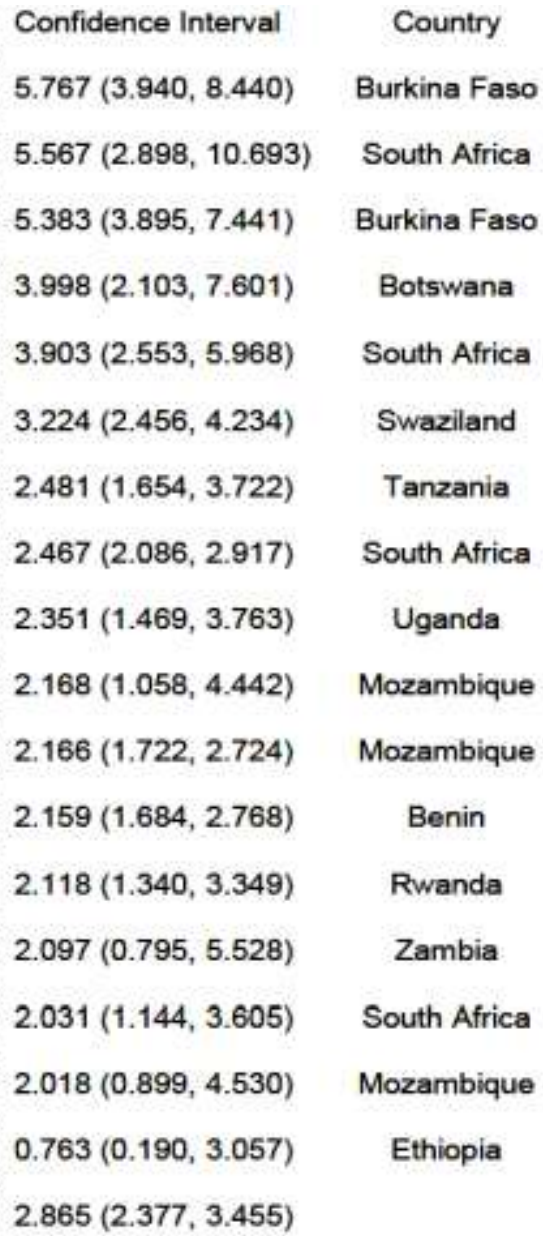

Country

Burkina Faso

South Africa

Burkina Faso

Botswana

South Africa

Swaziland

Tanzania

South Africa

Uganda

Mozambique

Mozambique

Benin

Rwanda

Zambia

South Africa

Mozambique

Ethiopia

Any anti-TB resistance in new TB cases

\begin{tabular}{cccc}
\hline \multicolumn{4}{c}{ Heterogeneity test } \\
\hline $\mathrm{I}^{2}$ & $\mathrm{Q}$ & $\mathrm{DF}$ & $\mathrm{P}$-val (Q) \\
$72.3 \%$ & 57.747 & 16.000 & 0.000 \\
\hline
\end{tabular}

Figure 4. Risk ratio of any anti-TB drug resistance in relation to previous history of anti-TB treatment (previously TB treated vs New TB cases), a meta-analysis in Sub-Saharan Africa. 
Previous Anti-TB Treatment and MDR TB: The risk of having MDR-TB in previously anti-TB treated individuals was more than five- fold higher than that of new TB cases (overall $\mathrm{RR}=5.7 ; 95 \%$ CI, 3.61 to 8.96). Accordingly, the association of MDR-TB with previous anti-TB drug treatment was even stronger than those who had at least one drug-resistant TB. Even if fourteen of the sixteen studies and the pooled value showed a statistically significant association of MDR-TB with previous history of anti-TB drug treatment, two studies from Mozambique $(14,22)$, one study from Zambia (31) and one study from Ethiopia (36) were exceptions. Additionally, heterogeneity testing uncovered a significant variation among the included studies $\left(\mathrm{I}^{2}=75 \%\right)$. But the sensitivity analysis showed the stability of the overall RR (Fig 5).
Study Name

Murray J et al study 1 (2000)

Kenyon A T (1999)

Sangaré L et al study 2 (2011)

Sangaré L et al study 1 (2010)

Mulenga $C$ et al (2010)

Lukoye D et al (2011)

van Halsema C L et al (2012)

Affolabi D et al (2007)

Sanchez-Padella E et al (2012)

Gudo PS (2011)

Chonde TM et al (2010)

Nunes E A et al (2005)

Umubyeyi AN et al (2007)

Cox H S et al (2010)

Bruchfeld J eta al (2002)

Mac-Arthur A et al (2001)

Overall

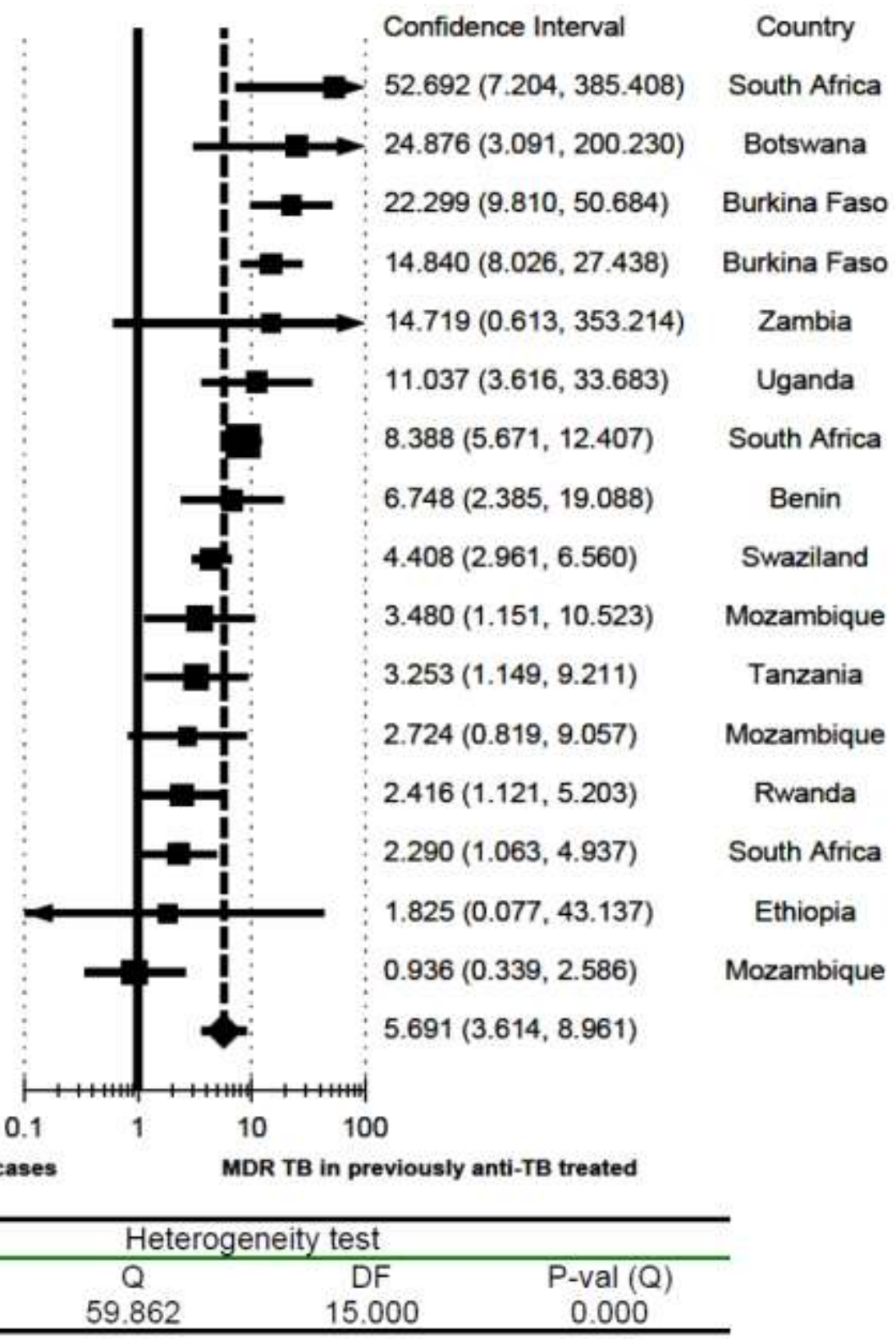

Figure 5. Risk ratio of multidrug resistant TB (MDR-TB) in relation to previous history of anti-TB treatment (previously TB treated vs New TB cases), a meta-analysis in Sub-Saharan Africa 
Resistance to First Line Anti-TB Drugs: Table 1 summarizes several meta-analyses done on the association of individual first line anti-TB drug resistance with anti-TB drug treatment history and HIV status. Statistically significant resistance was found in four of the first line anti-TB drugs among previously treated anti-TB drug treated individuals, but a very strong association was seen in ethambutol and rifampicin (the overall $\mathrm{RR}=5.8$ and 5.3, respectively). However, there was no increased risk of the selected first line anti-TB drug resistance by being HIV positive.

Table 1: Overall risk ratios of first line anti-TB drugs resistance in relation to previous history of anti-TB drug treatment or HIV status, a meta-analyses in Sub-Saharan Africa

\begin{tabular}{lllll}
\hline TB Resistance predictor & \multicolumn{2}{l}{ Risk of resistance } & \multicolumn{2}{c}{ Heterogeneity test } \\
\cline { 2 - 3 } & $\begin{array}{l}\text { Overall } \\
\text { Risk Ratio }\end{array}$ & $\mathbf{9 5 \%} \mathbf{C I}$ & $\mathbf{I}^{\mathbf{2}}$ & $\begin{array}{l}\text { Degrees of } \\
\text { freedom }\end{array}$ \\
\hline $\begin{array}{l}\text { By anti-TB treatment } \\
\text { history: }\end{array}$ & & & \\
$\quad$ Isoniazid & 3.22 & $2.45-4.24$ & $80 \%$ & 12 \\
$\quad$ Rifampicin & 5.25 & $3.27-8.37$ & $80 \%$ & 12 \\
$\quad$ Streptomycin & 2.68 & $1.89-3.82$ & $75 \%$ & 10 \\
$\quad$ Ethambutol & 5.81 & $3.35-10.05$ & $64 \%$ & 10 \\
By HIV status: & & & & \\
$\quad$ Isoniazid & 0.99 & $0.81-1.22$ & $0 \%$ & 4 \\
$\quad$ Rifampicin & 1.02 & $0.52-2.00$ & $55 \%$ & 3 \\
$\quad$ Streptomycin & 1.09 & $0.75-1.59$ & $42 \%$ & 3 \\
$\quad$ Ethambutol & 0.81 & $0.16-4.11$ & $52 \%$ & 3 \\
\hline
\end{tabular}

$\mathrm{TB}=$ Tuberculosis

\section{DISCUSSION}

This meta-analysis, which included mainly recently published studies, has demonstrated that the risk of developing single anti-TB drug resistance or MDR-TB was strongly associated with a previous history of exposure to anti-TB drugs, which is consistent with another metaanalysis that included studies conducted in the 1990s (21). A similar report also came from mainland China (8). The 2002-2007 global survey conducted by WHO also showed that the highest prevalence of MDR-TB among newly diagnosed and previously treated TB cases was $22 \%$ and $60 \%$, respectively (5). The consistent findings across decades in the majority of studies and in this meta-analysis probably indicate how much the Mycobacterium TB is predisposed to develop resistance when it is exposed to anti-TB drugs. This may be related to poor patient's adherence to treatment schedule, as previous reports showed (8).

However, several questions need to be answered before a cause and effect conclusion can be made. First, despite our extensive search of electronic databases for studies in the region, we found no studies that assessed the presence of vertical (resistance to the same drug exposed to before) or horizontal drug resistance (cross resistance developed after exposure to any of the other anti-TB drugs before) using the Mycobacterium TB strains database. In other words, had this evidence been found in studies that compared the sensitivity pattern of the TB strain for the first line drugs in the initial infection with the TB strain in the repeated infection, one could ascertain the direct cause of previous treatment for vertical and/or cross resistance in the included studies.

Otherwise, in some of the TB patients diseased for the second time, their previous treatment might have been counted wrongly as a risk factor for the developed resistance while the real story is that they were initially infected with a resistant TB strain. The same is true for reinfection: the initial infection might have been cured but the second time the patient could have acquired a resistant TB strain, in which the previous treatments was wrongly given credit for the development of drug resistance among reinfected patients. Similarly, the new TB cases, which were found to have a lesser degree of drug 
resistance, probably had an equal chance of acquiring resistant TB strains. Furthermore, this meta-analysis has shown that the association of MDR-TB with previous TB drug treatment was stronger than any anti-TB drug resistance, which probably indicates the possibility of acquiring resistant TB strains in the initial infection or the increased probability of cross resistance. It was also reported that patients infected by drugresistant $\mathrm{TB}$ and treated with short course chemotherapy are likely to acquire resistance through inadvertent monotherapy, the so called "amplifier effect" (42). This is very likely to happen in SSA as sensitivity testing is not routinely done before initiating short course antiTB therapy.

Second, the data set was also inadequate or not fit for meta-analysis in terms of the contribution of the anti-TB treatment pattern in the previous infection. Several of the studies compared the resistance of new TB and previously TB treated cases without further stratifying the initial treatment pattern by duration, adherence to anti-TB drugs, and defaulting from taking drugs, which were attributed as important risk factors for occurrence of resistance $(7,8)$.

In the authors' opinion, among others, establishing a Mycobacterium TB strains database at the regional level is a top priority. Such a database would definitely help in identifying the sensitivity pattern of initial and repeat TB infections, allowing the timely detection of resistant strains and the taking of appropriate actions. We guess such initiative will not be as costly as the second line therapy, the economic and social impact of increasingly spreading resistant TB strains in the region.

Some years back, it was reported that Mycobacterium TB is more liable to undergo mutation when it is exposed to isoniazid and rifampicin than to streptomycin and many of the second line anti-TB drugs (43). Although it was not possible to make analysis on comparisons of TB strains drug sensitivity in the initial and repeat infections, the resistance pattern between new and repeat infections with regard to the individual antiTB drugs demonstrated that the strength of resistance association with previous TB drug treatment was very high in ethambutol and rifampicin, and low in streptomycin. The proportion of resistance to isoniazid in primary studies was the highest in both new and previously anti-TB treated cases. As a result, the RR was falsely the second lowest. Such high proportion of resistance to isoniazid in both new and previously treated anti-TB cases was probably because of its wide use as prophylaxis in HIV positives. Similarly, TB resistance to isoniazid is the most common type in the United States (44).

Discussing the mechanism for the development of resistance for each drug is beyond the scope of this manuscript. However, when we look at the findings in the individual studies, fewer resistant cases were found with ethambutol in the new than in the previously TB treated cases (1517, 20, 23, 29, 31, 33, 39). Could its bacteriostatic nature (mycobacterial cell wall biosynthesis inhibition) explain why Mycobacterium TB strains became highly resistant to ethambutol among the previously treated? The challenge is that rifampicin (bactericidal and mycobacterial RNA polymerase enzyme inhibitor), which is thought to be the cornerstone of short-course chemotherapy, was almost equally ineffective in previously TB treated patients.

On the other hand, HIV infection is a known risk factor for increased incidence of TB disease, particularly in SSA where the burden of HIV infection is so high (2). In this meta-analysis, however, HIV co-infection was not found to have a statistically significant association with anti-TB drug resistance, which is consistent with other studies $(8,21)$. This is in contrast to a report from about two decades ago, which showed that $90 \%$ of cases of MDR-TB occurred in HIV-co-infected patients (45). Similarly, in the United States, it was reported that HIV infection was associated with MDR-TB (46). About fifteen years ago, Anastasis et al expressed concern regarding the development of drug-resistant TB as the HIV epidemic progressed (14). However, the current meta-analysis provides strong evidence to affirm that HIV co-infection may not have an influence on the development of anti-TB drug resistance. Additionally, with the exception of a report from Latvia and Ukraine, several reports from different parts of the world have shown that HIV infection has no statistically significant association with MDR-TB (47).

HIV is known to suppress the human cellular immunity and shorten the period from TB infection to TB disease (48); however, as this 
analysis showed, the chance of developing drugresistant TB seems not to be related with immunity. Even a study that compared a culture conversion with treatment among HIV-positives and negatives in MDR-TB patients revealed that there was no difference in the proportion who converted based on HIV status (49).

The core point needing to be underlined is that SSA (with the exception of some districts of South Africa) is characterized by a low prevalence of drug-resistant TB (5), and a high prevalence of both TB and HIV infections (3). Had HIV coinfection been a strong factor for occurrence of drug-resistant TB, SSA would have taken the lead. The implication is 1) Several of HIV infected patients in SSA didn't have a chance to acquire a drug-resistant Mycobacterium TB strain and 2) The mechanism for resistance development in Mycobacterium TB may not be related to the suppression of the host's cellular immunity as resistance is the microorganisms' self-defense mechanism.

It should be noted that we are not suggesting that HIV-infection has no role in the epidemiology of drug-resistant TB. Being HIV infected is a risk for developing TB disease and is equally a risk for being diseased with resistant TB and spreading it to others who are susceptible. Therefore, although this meta-analysis and others did not demonstrate statistically significant associations of drugresistant TB with HIV infection, it is our understanding that HIV has a significant role in fueling the spread of drug-resistant TB by accelerating the progression from $\mathrm{TB}$ infection to TB disease.

Some authors have referred to MDR-TB as "the third epidemic" (third to HIV and TB) to show the increasing magnitude of drug resistant TB across the world (50). This meta-analysis has also shown that previous anti-TB drug treatment had a strong association with the development of MDR-TB. One of the strengths of this metaanalysis is the finding regarding the resistance pattern of individual anti-TB drugs. The RRs of drug resistance among previously TB treated cases were significantly higher in ethambutol and rifampicin than others. However, HIV infection has not been associated with an increased risk of anti-TB drug resistance. Nevertheless, the high RR of MDR-TB, ethambutol, and rifampicin among previously $\mathrm{TB}$ treated cases are a big warning for this region, where the proportion of $\mathrm{TB}$ cases is very high (47). This situation warrants timely detecting and finding appropriate treatment for drug-resistant TB cases, which would help curtail transmission to many HIV positive individuals in the region.

As limitation, since most of the included studies didn't report the number of relapse cases, treatment failure cases, patients' ART status and HIV clinical stages, it was not possible to conduct meta-regression analysis and subgroup analysis to estimate their effect on the association of anti-TB treatment history and HIV infection with drug resistant TB.

This meta-analysis has demonstrated that the risk of developing single anti-TB drug resistance or MDR-TB was strongly associated with a previous history of exposure to anti-TB drugs, which needs urgent revision of prevention policyin the modality of preventing the occurrence and transmission of resistant TB strains. Furthermore, primary treatment needs to be given special emphasis, including sporadic screening for drug sensitivity before initiating treatment, and establishing a TB strains database for tracing and in-depth molecular studies. The direct observation therapy, short course program (DOTS), which is ascribed as one of the reasons for low drugresistant TB prevalence in SSA (47), has to be strengthened.

\section{ACKNOWLEDGMENT}

We would like to thank Dr. William Burton from Albert Einstein Medical College for editing this manuscript.

\section{REFERENCES}

1. World Health Organization (WHO): The stop TB department 2012. http://www.who.int/tb/publications/CORPORATE FACTSHEET_May2012.pdf

2. World Health Organization (WHO): Global Tuberculosis Control 2011. http://whqlibdoc.who.int/publications/2011/978924 1564380_eng.pdf

3. Mayer K H, Hamilton C H. Synergistic Pandemics: Confronting the Global HIV and Tuberculosis Epidemics. Clinical Infectious Diseases 2010; 50(S3):S67-S70.

4. World Health Organization (WHO): The evolving threat of antimicrobial resistance: options for 
action. 2012 http:// whqlibdoc.who.int/publications/2012/pdf

5. The WHO/IUATLD: Global Project on Antituberculosis Drug Resistance Surveillance 20022007. Anti-Tuberculosis drug resistance in the world: Report 4. http://www.who.int/tb/publications/2008/pdf

6. Deutschendorf C, Goldani L Z, Pires dos Santos R. Previous use of quinolones: a surrogate marker for first line anti-tuberculosis drugs resistance in HIVinfected patients? Braz J Infect Dis. 2012; 16(2):142-145.

7. van der Werf M J, Langendam M W, Huitric E, Manissero D. Multidrug resistance after inappropriate tuberculosis treatment: a metaanalysis. Eur Respir J 2012; 39: 1511-1519.

8. Zhao P, Li XJ, Zhang SF, Wang XS, Liu CY. Social behavior risk factors for drug resistance tuberculosis in main land china: a meta-analysis. J Int Med Res 2012; 40:436-445.

9. Blöndal K. Barriers to reaching the targets for tuberculosis control: multidrug-resistant tuberculosis. B World Health Organ 2007; 85: 387 394.

10. Haar C H, Cobelens FGJ, Kalisvaart NA, van der Have JJ, van Gerven PJHJ, van Soolingen D. Tuberculosis Drug Resistance and HIV Infection, the Netherlands. Emerg Infect Dis 2007; 13 (5): $776-778$.

11. Yimer SA, Agonafir M, Derese Y, Sani Y, Bjune GA, Holm-Hansen C. Primary drug resistance to anti-tuberculosis drugs in major towns of Amhara region, Ethiopia. APMIS 2012; 120: 503-509.

12. Demissie M, Lemma E, Gebeyehu M, Lindtjorn B. Sensitivity to Anti tuberculosis Drugs in HIVpositive and negative Patients in Addis Ababa. Scand J Infect Dis 2001; 33: 914-919.

13. van Halsema CL, Fielding KL, Chihota VN, Lewis JJ, Churchyard GJ,Grant AD. Trends in drugresistant tuberculosis in a gold-mining workforce in South Africa, 2002-2008. Int J Tuberc Lung Dis 2012; 16(7): 967-973.

14. Anastasis D, Pillai G, Rambiritch V, Karim SSA. A retrospective study of human immunodeficiency virus infection and drug-resistant tuberculosis in Durban, South Africa. Int J Tuberc Lung Dis 1997; 1 (3): 220-224.

15. Affolabi D, Adjagba OABG, Tanimomo-Kledjo B, Gninafon M, Anagonou SY, Portaels F. Antituberculosis drug resistance among new and previously treated pulmonary tuberculosis patients in Cotonou, Benin. Int J Tuberc Lung Dis 2007; 11(11):1221-1224.

16. Sangaré L, Diandé S, Badoum G, Dingtoumda B, Traoré AS. Anti-tuberculosis drug resistance in new and previously treated pulmonary tuberculosis cases in Burkina Faso. Int J Tuberc Lung Dis 2010; 14(11):1424-1429 (Study 1).

17. Mac-Arthur A, Gloyd S, Perdigão P, Noya A, Sacarlal J, Kreiss J. Characteristics of drug resistance and HIV among tuberculosis patients in Mozambique. Int J Tuberc Lung Dis 2001; 5(10): 894-902.

18. Diandé S, Sangaré L, Kouanda S, Dingtoumda BI, Traoré AS. Drug Resistance of Mycobacterium tuberculosis Complex among Newly Diagnosed Tuberculosis Cases in Burkina Faso. WAJM 2009; 28 (6): 353-357.

19. Bercion $\mathrm{R}$, Kuaban C. Initial resistance to antituberculosis drugs in Yaounde, Cameroon in 1995. Int J Tuberc Lung Dis 1997; 1 (2): 110-114.

20. Lukoye D, Cobelens FGJ, Ezati N, et al. Rates of Anti-Tuberculosis Drug Resistance in KampalaUganda Are Low and Not Associated with HIV Infection. PLoS ONE 2011; 6(1): e16130. doi:10.1371/journal.pone.0016130.

21. Suchindran S, Brouwer ES, Van Rie A. Is HIV Infection a Risk Factor for Multi-Drug Resistant Tuberculosis? A Systematic Review. PLoS ONE 2009; 4(5):e5561. doi:10.1371/journal.pone.0005561

22. Nunes EA, De Capitani EM, Coelho E et al. Patterns of anti-tuberculosis drug resistance among HIV-infected patients in Maputo, Mozambique, 2002-2003. Int J Tuberc Lung Dis 2005; 9(5):494500.

23. Sanchez-Padilla ES, Dlamini T, Ascorra A, et al. High prevalence of multidrug resistant tuberculosis, Swaziland, 2009-2010. Emerg Infect Dis 2012; 18(1): 29-37.

24. Davies GR, Connolly C, Sturn AW, McAdam KPWJ, Wilkinson D. Twice-Weekly, directly observed treatment for HIV infected and uninfected tuberculosis patients: cohort study in rural South Africa. AIDS 199; 13: 811-817.

25. Minime-Lingoupou F, Manirakiza A, Yango F, Zandanga G, Le Faou A, Rigouts L. Relatively low primary resistance to anti-tuberculosis drugs in Bangui and Bimbo, Central African Republic. Int J Tuberc Lung Dis 2011; 15(5): 657-661.

26. Abebe G, Abdissa K, Abdissa A et al. Relatively low primary drug resistant tuberculosis in Southwestern Ethiopia. BMC Res Notes 2012; 5: e225. www.biomedcentral.com/1756-0500/5/225.

27. Urassa W, Mugusi F, Villamor E et al. Primary antimicrobial resistance among mycobacterium tuberculosis isolates from HIV seropositive and HIV seronegative patients in Dar es Salaam, Tanzania. BMC Res Notes 2008; 1:e58. www.biomedcentral.com/1756-0500/1/58 
28. Lawson L, Habib AG, Okobi MI et al. Pilot study on multidrug resistant tuberculosis in Nigeria. Ann Afr Med 2010; 9(3): 184-187.

29. Chonde TM, Basra D, Mfinanga SGM et al. National anti-tuberculosis drug resistance study in Tanzania. Int J Tuberc Lung Dis 2010; 14(8): 967972.

30. Asiimwe BB, Ghebremichael S, Kallenius G, Koivula T, Joloba MS. Mycobacterium tuberculosis spoligotypes and drug susceptibility pattern of isolates from tuberculosis patients in peri-urban Kampala, Uganda. BMC infect Dis 2008; 8:e101. www.biomedcentral.com/14712334/8/101.

31. Mulenga C, Chonde A, Bwalya IC et al. Low occurrence of tuberculosis drug resistance among pulmonary tuberculosis patients from urban setting with a long running DOTS program in Zambia. Tuberc Res Treat 2010; doi:10.1155/2010/938178

32. Kenyon TA, Mwasakaga MJ, Huebner R, Rumisha D, Binkin N, Maganu E. Low levels of drug resistance amidst rapidly increasing tuberculosis and human immunodeficiency virus co-epidemics in Botswana. Int J Tuberc Lung Dis 1999; 3(1): 411.

33. Gudo PS, Cuna Z, Coelho E et al. Is MDR-TB on the rise in Mozambique? Results of a national drug resistant survey. Eur Respir J 2011; 38(1): 222225.

34. Murray J, Sonnenberg P, Shearer SC, GodfreyFaussett. Human immunodeficiency virus and the outcome of treatment for new and recurrent pulmonary tuberculosis in African patients. Am J Respir Crit Care Med 1999; 159:733-740 (study 2).

35. Sangare L, Diande S, Ouedrogo G, Traore AS. HIV infection and mycobacterium tuberculosis drug resistance among tuberculosis patients in Burkina Faso, West Africa. Afr.J.Cln.Exper.Microbiol 2011； 12(1): 38-43 (study 2).

36. Bruchfeld J, Aderaye G, Palme IB et al. Evaluation of outpatient with suspected pulmonary tuberculosis in high HIV prevalent setting in Ethiopia: clinical, diagnostic and epidemiological characteristics. Scand J Infect Dis 2002; 34: 331337.

37. Cox HS, McDermid C, Azevedo V et al. Epidemic levels of drug resistance tuberculosis (MDR and XDR-TB) in a high HIV prevalence setting in Khayelitsha, South Africa. PLoS ONE 2010; 5(11): e13901.doi:10.1371/journal.pone.0013901.

38. Calver AD, Falmer AA, Murray $M$ et al. Emergence of increased resistance and extensively drug resistant tuberculosis despite treatment adherence, South Africa. Emerg Infect Dis 2010; 16(2): 264-271.

39. Murray J, Sonnenberg P, Shearer SC, GodfreyFaussett. Drug-resistant pulmonary tuberculosis in a cohort of South African gold miners with a high prevalence of HIV infection. S Afr Med J 2000; 90: 381-386 (study 1).

40. Umubyeyi AN, Rigouts L, Zissis G et al. Primary and acquired resistance to anti-tuberculosis in strains of mycobacterium tuberculosis isolated in Rwanda. Med Trop 2007; 67: 149-153.

41. Bazira J, Asiimwe BB, Joloba ML, Bwanga F, Matee MI. Use of the GenoType MTBDRplus assay to assess drug resistance of Mycobacterium tuberculosis isolates from patients in rural Uganda. BMC Clinical Pathology 2010, 10:5 http://www.biomedcentral.com/1472-6890/10/5

42. Farmer $\mathrm{P}$, Bayona J, Becerra $\mathrm{M}$ et al. The dilemma of MDR-TB in the global era. Int J Tuberc Lung Dis 1998; 2: 869-876.

43. Ramaswamy S, Musser JM. Molecular genetic basis of antimicrobial agent resistance in Mycobacterium tuberculosis: 1998 update. Tuber Lung Dis. 1998; 79: 3-29.

44. CDC. Reported Tuberculosis in the United States, 2006, Atlanta. www.cdc.gov/tb/surv2006/

45. Snider Jr DE, Roper WL. The new tuberculosis. N Engl J Med 1992; 326: 703-705.

46. Frieden TR, Sterling T, Pablos-Mendez A, Kilburn JO, Cauthen GM, Dooley SW. The emergence of drug-resistant tuberculosis in New York City. N Engl J Med 1993; 328(8): 521-526.

47. Getahun H, Gunneberg C, Granich R, Nunn P. HIV infection-associated tuberculosis: The epidemiology and the response. Clin Infect Dis 2010; 50(S3): S201-S207.

48. Evans Amukoye. Multidrug resistant tuberculosis: a challenge in the management of tuberculosis. Afr J Health Sci 2008; 15:6-13.

49. Brust JCM, Lygizos M, Chaiyachati $\mathrm{K}$ et al. Culture conversion among HIV co-infected multidrug resistant tuberculosis patients in Tugela Ferry, South Africa. PLoS ONE 2011; 6(1): e15841

50. Nevil K, Bromberg A, Bromberg R, Bonk S, Hanna BA, Rom WN. The third epidemicmultidrug-resistant tuberculosis. Chest 1994; 105(1): 45-48. 